\title{
The Automatic Detection of Near-Earth Asteroids in Co-orbital State with Terrestrial Planets by Implementing Motif Discovery Algorithm
}

\author{
Judhistira Aria Utama ${ }^{1}$, Lala Septem Riza $^{2}$, Muhammad Naufal Fazanadi ${ }^{3}$, Taufiq Hidayat ${ }^{4}$ \\ \{j.aria.utama@upi.edu'1 , lala.s.riza@upi.edu², mnf_naufal@student.upi.edu ${ }^{3}$, taufiq@as.itb.ac.id $\left.{ }^{4}\right\}$ \\ Department of Physics Education Universitas Pendidikan Indonesia, Jl. Dr. Setiabudhi 229 Bandung \\ 40154, Jawa Barat, Indonesia ${ }^{1}$, Department of Computer Science Education Universitas Pendidikan \\ Indonesia, J1. Dr. Setiabudhi 229 Bandung 40154, Jawa Barat, Indonesia ${ }^{2,3}$, Astronomy Research \\ Division, Institut Teknologi Bandung, Jl. Ganesha 10 Bandung 40132, Jawa Barat, Indonesia ${ }^{4}$
}

\begin{abstract}
Orbital computation of 3,372 real near-Earth asteroids (NEAs) population was conducted and their evolution was recorded every 1,000 years for the next 5 Megayears (Myr). This study is aimed to detect asteroids in 1:1 mean-motion resonance (MMR) with planets. In order to achieve our goal, we employed N-body numerical simulation with the use of Swift-RMVS (Regularized Mixed-Variable Symplectic) integrator. We found that NEAs population could have evolved into asteroids in a co-orbital state with Terrestrial planets (Mercury to Mars). In order to detect this co-orbital condition, the time-series motif discovery algorithm was applied against asteroids' orbital element. The predicted motif location and duration of 1:1 MMR were then compared with one of the standard software in astronomy that showed conformity of results greater than $80 \%$.
\end{abstract}

Keywords: Co-orbital state, Mean-motion resonance, Motif discovery, Near-earth asteroids, Orbital elements.

\section{Introduction}

By definition near-Earth asteroids (NEAs) is a population of asteroids that have the closest orbital distance to the Sun (perihelion, $q$ ) of less than 1.3 astronomical units (au; 1 au is the Earth's average distance from the Sun. According to International Astronomical Union in 2012, 1 au equals $1.495978707 \times 10^{11}$ meters exactly) and the farthest orbital distance from the Sun (aphelion, $Q$ ) of greater than 0.983 au. We called asteroids are in mean-motion resonance (MMR) with a particular planet when the asteroid's motion is coupled to the motion of a planet in such a way that strengthens the influence of the planet on the asteroid. The resonance means that the perihelions of the two objects are nearly aligned and conjunctions occur when a planet and an asteroid are near perihelion. The MMR exists at semi-major axes (the object's average distance from the Sun, $a$ ) where the period of the asteroid and the planet form a ratio of small integers, $r: p$ and resonant angle $\phi_{r p}=r \lambda_{\text {asteroid }}-p \lambda_{\text {planet }}-(r-p) \varpi_{\text {asteroid. }}$. The symbol $\lambda$ and $\varpi$ are mean longitude and longitude of perihelion, respectively. In the special case of $r=p$, this condition is known as co-orbital. Thus, co-orbital asteroids are those in 1:1 MMR with a planet and a librating or oscillating resonant angle $\phi_{11}=\lambda_{\text {asteroid }}-\lambda_{\text {planet }}$ around an equilibrium value. For this special case, the resonant angle $\phi_{11}$ librates around one of four values: $0^{\circ}, 60^{\circ}, 180^{\circ}$ and 
$300^{0}[1]$. In such conditions, asteroids have an orbital period very similar to the planet 's orbital period.

Asteroids in 1:1 MMR with a planet can be in stable co-orbital (they are on long-term stable orbit for the age of the solar system) or temporary co-orbital (leave the co-orbital orbit on significantly shorter time scale than the age of the solar system). Of the three types of orbit in a co-orbital state, i.e. quasi-satellite, tadpole and horseshoe [1][2], only asteroids with tadpole orbit are generally staying in long-term stability [1][3]. The study of the existence of objects in the 1:1 MMR state with Terrestrial planets through a numerical simulation back to the work initiated by Christou [4]. Morais \& Morbidelli [5][6] have estimated the number of NEAs population those are in a co-orbital state with Earth and Venus. The co-orbital asteroids have also the potential to become targets for non-Terrestrial mining of raw materials on unmanned missions [7]. To determine whether asteroids are co-orbital, one needs to compute the resonance variables during the investigated time span for every object, therefore this task can be considered as computational workload [8]. In order to make identification of 1:1 MMR easier, we present an automated method implementing motif discovery algorithm [9][10][11] employed on orbital elements of an asteroid and the Terrestrial planet considered. In our case, by utilizing motif discovery algorithm the pattern produced from a co-orbital motion of an asteroid and a planet can be recognized without any visual inspection on the object's orbital element time-series data.

\section{Method and Data Processing}

Asteroid's orbital elements data required in our study was obtained from JPL-NASA Small Body Database Search Engine ${ }^{1}$. By filtering only for NEAs of the four main classes (Amor, Apollo, Aten and Atira) with very well known orbits (which corresponds to the orbit error limit less than 1 arcsecond/decade) according to epoch MJD57400, we have successfully selected the 3,372 asteroids.

We followed the evolution of our asteroids sample by employing RMVS (Regularized Mixed-Variable Symplectic) integration method included in Swift integrator package [12] for 5 Megayears (Myr) in the future as they became co-orbital with the Terrestrial planets and until they collide with the Sun or planets or achieve $a>100$ au (at which point we consider asteroids as were ejected out of the solar system). The orbital elements of asteroids were recorded every 1,000 years in the form of a binary file (consisting of the object's identity, time, and six orbital elements of the object) that can be read with software such as SwiftVis ${ }^{2}$. With the help of SwiftVis, the binary file can be converted into a file with extension *.txt.

In implementing the time-series motif discovery algorithm, we designed a standalone Python computation model. The process begins by providing input (i.e. objects orbital elements) into the program for further pre-processing, data normalization, converting into SAX (Symbolic Aggregate Approximation) time-series, motif recognition with random projection and postprocessing. The SAX algorihm was used to make the matching data process easier by converting the real number of time-series data into discrete time-series. To find a similar pattern found in the discrete time-series data we implemented the random projection algorithm. The example of the motif to be found is shown in Figure 1. Because we focus on finding the location, time of occurrence and duration of 1:1 MMR, we only made use of the object's identity, time ( $t$, in day

\footnotetext{
${ }^{1} \mathrm{http}: / /$ ssd.jpl.nasa.gov/sbdb_query.cgi

${ }^{2}$ https://www.cs.trinity.edu/ mlewis/SwiftVis/
} 
unit) and semi-major axes ( $a$, in au) information. After the motif was found in the time-series of $a$ for an asteroid, further testing was carried out whether at that location did occur 1:1 MMR between the asteroid and a particular Terrestrial planet. In this step, the asteroid's mean value of $a$ over the motif-discovered time span was then calculated and compared with the value of $a$ for a given planet by using a threshold of $\Delta a=0.05$ au and minimal duration of a co-orbital motion $\Delta t=10,000$ years.

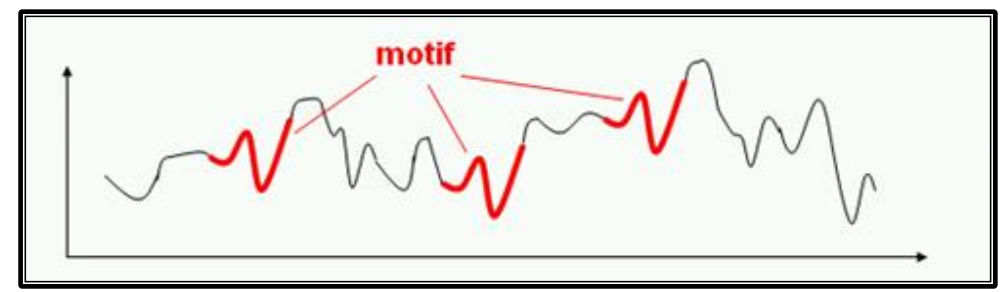

Fig. 1. Example of time-series data containing 3 identical subsequences.

\section{Results}

Figure 2 below shows the $a$ values as a function of time of an asteroid with particleID = 3 as well as the locations of 1:1 MMR found by our standalone python computation model.

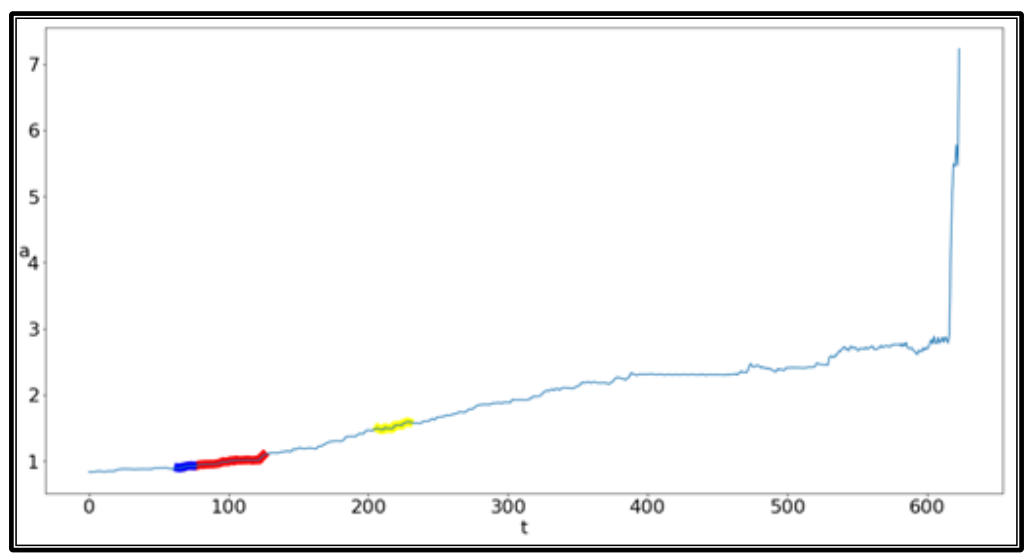

Fig. 2. The location of 1:1 MMR of an asteroid with particleID = 3 with the Moon (blue color), with the Earth (red color) and with planet Mars (yellow color). The $\mathrm{X}$-axis is time in year ( $\times 1,000$ years) while $\mathrm{Y}$-axis is asteroid's semi-major axes in au.

In our solar system model implemented in the integrator package, the Moon was treated as a separated object instead of as the Earth's natural satellite. We found that asteroid with particleID $=3$ trapped in co-orbital motion with the Moon for 11,000 years, with the Earth as long as 56,000 years and with planet Mars for 30,000 years. The detection of 1:1 MMR between an asteroid and a planet through the implementation of motif discovery algorithm that we have done will be compared then with the results of software SwiftVis. 
For comparison purpose, we took 30 asteroids of 3,372 NEAs population used in our integration. All co-orbital time span of our results that intersect among them is combined and then compared with the results from SwiftVis. For example, an asteroid with particleID $=6$ was in a co-orbital motion with planet Mars two times, i.e. within a time span of 71,000 years 82,000 years and 76,000 years $-86,000$ years. Because both time span has an intersection from 76,000 to 82,000 , they will be combined into a new time span of 71,000 years $-86,000$ years. Then this new time span is compared with the output of SwiftVis, which is 71,000 years 86,000 years. Thus for this asteroid, it is said that the resonance determination that occurs with a particular planet, as well as its duration, has $100 \%$ accuracy since it matches the result provided by SwiftVis.

We have also found that our results differ significantly from SwiftVis. It is shown on an asteroid with particleID $=7$. Our model predicts that there is no co-orbital motion that belongs to this asteroid with any particular planet but according to SwiftVis the asteroid experienced 1:1 MMR with planet Mars for 23,000 years. The difference can arise from the use of asteroid's mean semi-major axes value during the motif-discovered time span in our model while SwiftVis used actual asteroid's semimajor axes value. By using asteroid's mean value of $a$ as our reference, it could be that the value of $a$ found in the motif is out of range of ( $a_{\text {planet }} \pm 0.05 \mathrm{au}$ ) so that it is concluded that there are no 1:1 MMR between an asteroid with a particular planet. From the results of 30 sample asteroids, we obtained that the conformity of our model with SwiftVis reached $>80 \%$.

\section{Conclusions}

This work managed to design a computation model on the standalone Python for motif discovery by using the SAX and random projection algorithms implemented on the recognition of the 1:1 MMR pattern between an asteroid and a particular planet. The mean accuracy achieved by our model when compared with standard software SwiftVis reaches $>80 \%$.

Acknowledgments. This research is funded by the Ministry of Research, Technology, and Higher Education of the Republic of Indonesia under contract No. 171A/UN40. D/PP/2019.

\section{References}

[1] Mikkola, S., Innanen, K., Wiegert, P., Connors, M., and Brasser, R.: Stability limits for the quasisatellite orbit. Monthly Notices of Royal Astronomical Society. pp. 15-24 (2006)

[2] Murray, D. and Dermott, S.F.: Solar system dynamics. pp. 97-102. Cambridge University Press, US (2005)

[3] Ćuk, M., Hamilton, D.P., and Holman, M.J.: Long-term stability of horseshoe orbits. Monthly Notices of Royal Astronomical Society. pp. 3051-3056 (2012)

[4] Christou, A.A.: A numerical survey of transient co-orbitals of the terrestrial planets. Icarus. pp. 120 (2000)

[5] Morais, M.H.M. and Morbidelli, A.: The population of near-earth asteroids in coorbital motion with the earth. Icarus. pp. 1-9 (2002)

[6] Morais, M.H.M. and Morbidelli, A.: The population of near-earth asteroids in coorbital motion with the venus. Icarus. pp. 29-38 (2006)

[7] Ross, S.D.: Near-earth asteroid mining. Space Industry Report. pp. 1-24 (2001) 
[8] Forgács-Dajka, E., Sándor, Zs., and Érdi, B.: A fast method to identify mean motion resonances. Monthly Notices of Royal Astronomical Society. pp. 3383-3389 (2018)

[9] Lin, J., Keogh, E., Lonardi, S., and Patel, P.: Finding motifs in time-series. Proc. of the 2nd Workshop on Temporal Data Mining. pp. 53-68 (2002)

[10] Mueen, A., Keogh, E., Zhu, Q., Cash, S., and Westover, B.: Exact discovery of time-series motifs. Proceedings of the 2009 SIAM International Conference on Data Mining. pp. 473-484 (2009)

[11] Mueen, A., Keogh, E., and Bigdeli-Shamlo, N.: Finding time-series motifs in disk-resident data. Proceedings of the 2009 Ninth IEEE International Conference on Data Mining. pp. 367-376 (2009)

[12] Levison, H.F. and Duncan, M.J.: The long-term dynamical behavior of short-period comets. Icarus. pp.18-36 (1994) 